\title{
EXAME PAPANICOLAOU NA PREVENÇÃO DO CÂNCER NO COLO UTERINO: UMA REVISÃO INTEGRATIVA
}

\author{
PAPANICOLAOU EXPERIMENT IN THE PREVENTION OF CANCER IN \\ THE
}

UTERINE COLUMN: AN INTEGRATING REVIEW

\begin{abstract}
Kássia Camila Camargo de Sá (SÁ, K. C. C.) Curso de Biomedicina. Faculdade Evangélica de Ceres, Ceres - GO, Brasil. katinha_lucas@hotmail.com
\end{abstract}

Luciano Ribeiro Silva (SILVA, L. R.) Especialista em Citologia Clínica, Docente da

Faculdade Evangélica de Ceres, Ceres - GO, Brasil. luciano_rsilva@ig.com.br

Endereço para correspondência: Av. Brasil, s/n - Setor Morada Verde, Ceres - GO. Brasil. CEP: 76300-000 e-mail: katinha_lucas@hotmail.com

\section{RESUMO}

INTRODUÇÃO: A alta incidência do câncer uterino é resultado da exposição aos vários fatores de risco, tais como: o Papilomavírus humano (HPV), o tabagismo, as Infecções Sexualmente Transmissíveis (IST), o início precoce das relações sexuais, é uma doença com alto índice de prevenção, que possui um método efetivo para seu rastreamento, o exame Papanicolaou. Através da realização deste exame de prevenção é possível reduzir em até $70 \%$ a mortalidade por esse tipo de câncer na população de risco, pois o câncer de colo uterino tem um desenvolvimento lento. O objetivo principal foi destacar a importância da realização do exame citopatológico como o principal método de diagnóstico precoce do câncer no colo uterino. METODOLOGIA: Trata-se de uma revisão integrativa da literatura, com o delineamento de pesquisa bibliográfica em bases de dados virtuais Scientific Electronic Library Online (SCIELO), Instituto Nacional do Câncer (INCA) e Biblioteca Virtual de Saúde (BVS). Como critério de inclusão para a seleção do material, definiu-se a utilização de artigos científicos nacionais, de fontes fidedignas e de relevância sobre $\mathrm{o}$ tema. RESULTADO E DISCUSSÃO: Foram selecionados 15 artigos. Após leitura criteriosa encontrou-se os seguintes dados: $26 \%$ dos artigos relatam sobre a importância da realização do exame Papanicolaou; $20 \%$ sobre a realização da coleta para o exame, finalidade do exame e sua sensibilidade; $27 \%$ apontam os fatores de risco e $27 \%$ dos artigos selecionados têm em comum a importância da realização do exame Papanicolaou e os fatores de risco. CONCLUSÃO: Por meio desta pesquisa foi possível destacar a importância da realização periódica do exame Papanicolaou na prevenção do câncer de colo do útero. Bem como a 
importância do profissional da saúde tenha conhecimentos suficientes em relação à neoplasia do colo do útero, tanto em relação aos fatores de risco, importância do exame e qualidade do material coletado para realização do mesmo, no diagnóstico precoce.

Palavras-Chave: Câncer de colo uterino. Fatores de risco. Exame Papanicolaou. Prevenção. Diagnóstico.

\begin{abstract}
INTRODUCTION: The high incidence of uterine cancer is a result of exposure to various risk factors such as: human papillomavirus (HPV), smoking, sexually transmitted infections (STI), early onset of sex, is a disease with high prevention rate, which has an effective method for its screening, the Pap smear. By conducting this screening test it is possible to reduce mortality by this type of cancer by up to $70 \%$ in the population at risk, because cervical cancer has a slow development. The main objective was to highlight the importance of cytopathological examination as the main method for early diagnosis of cervical cancer.

METHODOLOGY: This is an integrative literature review, with the design of bibliographic research in virtual databases Scientific Electronic Library Online (SCIELO), National Cancer Institute (INCA) and Virtual Health Library (VHL). As inclusion criteria for the selection of the material, it was defined the use of national scientific articles, reliable sources and relevance on the subject. RESULTS AND DISCUSSION: 15 articles were selected. After careful reading, the following data were found: $26 \%$ of articles report on the importance of performing the Pap smear; 20\% about the collection for the exam, purpose of the exam and its sensitivity; $27 \%$ indicate the risk factors and $27 \%$ of the selected articles have in common the importance of the Pap smear and the risk factors. CONCLUSION: Through this research it was possible to highlight the importance of periodic Pap smears in the prevention of cervical cancer. As well as the importance of health professionals have sufficient knowledge in relation to cervical cancer, both in relation to risk factors, importance of the examination and quality of the material collected to perform it, in the early diagnosis.
\end{abstract}

Keywords: Cancer of the uterine cervix. Risk factors. Papanicolaou exam. Prevention. Diagnostic. 


\section{INTRODUÇÃO}

No Brasil, o controle do câncer do colo do útero teve seu ponto de partida em iniciativas pioneiras de profissionais que trouxeram para nosso meio a citologia e a colposcopia, a partir dos anos 1940. O Presidente Juscelino Kubitschek patrocinou a construção, em 1956, do Centro de Pesquisas Luíza Gomes de Lemos, da Fundação das Pioneiras Sociais, no Rio de Janeiro - atualmente integrado ao INCA - para atender aos casos de câncer de mama e aparelho genital feminino, para o controle do câncer do colo do útero em nosso país. Entre 1972 e 1975, o Ministério da Saúde, por meio de sua recém-instituída Divisão Nacional de Câncer, desenvolveu e implementou o Programa Nacional de Controle do Câncer. Esta foi a primeira ação de âmbito nacional do Ministério da Saúde para a prevenção do câncer do colo do útero. Em 1984, foi implantado o Programa de Atenção Integral à Saúde da Mulher (PAISM). Em 1986, foi constituído o Programa de Oncologia (PRO-ONCO) que elaborou o projeto Expansão da Prevenção e Controle do Câncer Cervicouterino, identificando as ações necessárias para a expansão do controle dessa neoplasia, em 1988, conhecida por Consenso sobre a Periodicidade e Faixa Etária no Exame de Prevenção do Câncer Cervicouterino (Brasil 2016).

O câncer do colo uterino é considerado um importante problema de saúde pública nos países em desenvolvimento. Em relação a outras neoplasias, o câncer uterino é altamente prevenível e de evolução lenta, dispondo de exame de rastreamento simples, barato e eficaz na sua detecção. A incidência dessa doença está relacionada à exposição de fatores de risco e a não realização do exame. Neste sentido, a citologia oncótica tem demonstrado ser útil em reduzir a incidência e a mortalidade pelo câncer uterino (SOARES; SILVA, 2016).

Quadro 1 - Nomenclatura e Classificação

\begin{tabular}{|c|l|l|l|l|}
\hline $\begin{array}{l}\text { Classificação } \\
\text { citológica de de } \\
\begin{array}{l}\text { Papanicolaou } \\
(1941)\end{array}\end{array}$ & $\begin{array}{l}\text { Classificação } \\
\text { histológica da } \\
\text { OMS (1952) }\end{array}$ & $\begin{array}{l}\text { Classificação } \\
\text { histológica de } \\
\text { Richart (1967) }\end{array}$ & $\begin{array}{l}\text { Sistema } \\
\text { Bethesda (2001) }\end{array}$ & $\begin{array}{l}\text { Classificação } \\
\text { Citológica } \\
\text { Brasileira } \\
(2006)\end{array}$ \\
\hline Classe I & - & - & - & - \\
\hline Classe II & - & - & $\begin{array}{c}\text { Alterações } \\
\text { benignas }\end{array}$ & $\begin{array}{c}\text { Alterações } \\
\text { benignas }\end{array}$ \\
\hline- & - & - & $\begin{array}{c}\text { Atipias de } \\
\text { significado } \\
\text { indeterminado }\end{array}$ & $\begin{array}{c}\text { Atipias de } \\
\text { significado } \\
\text { indeterminado }\end{array}$ \\
\hline
\end{tabular}




\begin{tabular}{|c|c|c|c|c|}
\hline Classe III & $\begin{array}{c}\text { Displasia leve } \\
\text { Displasia } \\
\text { moderada e } \\
\text { acentuada }\end{array}$ & $\begin{array}{c}\text { NIC I } \\
\text { NIC II e }\end{array}$ & HSIL & LSIL \\
\hline Classe IV & $\begin{array}{c}\text { Carcinoma in } \\
\text { situ }\end{array}$ & NIC III & HSIL \\
Classe V & $\begin{array}{c}\text { Carcinoma } \\
\text { invasor }\end{array}$ & $\begin{array}{c}\text { Carcinoma } \\
\text { invasor } \\
\text { in situ (AIS) }\end{array}$ & $\begin{array}{c}\text { Carcinoma } \\
\text { invasor }\end{array}$ & HSIL \\
\hline
\end{tabular}

Fonte: BRASIL 2016

A alta incidência do câncer uterino é resultado da exposição aos vários fatores de risco, tais como: o Papilomavírus humano (HPV), o tabagismo, as Infecções Sexualmente Transmissíveis (IST), o início precoce das relações sexuais, o baixo nível socioeconômico e escolar, a multiparidade, a baixa ingestão de vitaminas, o uso contínuo de contraceptivos orais, a multiplicidade de parceiros e a imunossupressão (SILVA et al., 2014).

O câncer de colo do útero é uma doença com alto índice de prevenção, que possui um método efetivo para seu rastreamento, o exame Papanicolaou. Através da realização deste exame de prevenção é possível reduzir em até $70 \%$ a mortalidade por esse tipo de câncer na população de risco, pois o câncer de colo uterino tem um desenvolvimento lento, e as alterações celulares e lesões percursoras são facilmente descobertas no exame Papanicolaou (PINHEIRO et al., 2013).

Segundo o Instituto Nacional do Câncer (INCA) o câncer do colo uterino é o terceiro tumor maligno mais frequente na população feminina, e a quarta causa de morte de mulheres por essa neoplasia no Brasil. Para o Brasil, estão previstos 16.370 novos casos de câncer no colo uterino para cada ano do biênio 2018-2019, com um risco estimado de 15,43 casos a cada 100 mil mulheres (INCA, 2017). Com base nesses dados, vê-se a relevância do tema para a saúde da mulher, sendo possível através desse artigo informar a população feminina da importância da realização e seguimento do exame Papanicolaou.

Assim, este estudo teve como objetivo destacar a importância da realização do exame citopatológico como o principal método de diagnóstico precoce do câncer no colo uterino, bem como reforçar o papel do profissional da saúde no diagnósticos precoce e prevenção, demostrando o benefício da prevenção ou até mesmo no diagnóstico precoce uma possibilidade da cura aos pacientes. 


\section{METODOLOGIA}

Trata-se de uma revisão integrativa da literatura, sendo possível realizar pesquisas empíricas ou de literatura teórica, também permite a inclusão de estudos experimentais e nãoexperimentais (SOUZA, SILVA e CARVALHO, 2010).

Para nortear a presente revisão integrativa, formulou-se a seguinte questão: Com a realização do exame Papanicolaou é possível identificar, precocemente, o câncer do colo uterino, e reduzir a sua incidência?

O levantamento bibliográfico foi realizado nas bases de dados: Scientific Eletronic Library Online (Scielo), Instituto Nacional de Câncer (INCA), Biblioteca Virtual em Saúde

(BVS). Para escolha dos artigos, os descritores utilizados foram: "Câncer de colo uterino", "Fatores de risco", "Exame Papanicolaou", "Prevenção" e "Diagnóstico".

Para o refinamento adequado da pesquisa os critérios de inclusão utilizados foram estudos que abordassem a importância da realização do exame Papanicolaou como método de diagnóstico precoce e preventivo do câncer do colo uterino, como também os fatores influenciantes para o desenvolvimento dessa neoplasia. A busca foi somente de artigos de produção nacional publicados entre os anos de 2001 a 2017. Portanto, os artigos que não eram adequados aos critérios de inclusão foram enquadrados nos critérios de exclusão, sendo artigos de língua estrangeira, que não versassem sobre o tema do estudo e que antecedem o período estipulado para a pesquisa.

$\mathrm{Na}$ busca por meio dos descritores mencionados, foram encontrados 65 artigos que foram lidos na íntegra. Dos 65 artigos, 50 foram excluídos por não se adequarem ao definido para composição do artigo. Sendo assim, a amostra final foi composta por 15 artigos científicos. A partir dos artigos selecionados, o estudo foi dividido em duas etapas: na $1^{\circ}$ etapa realizou-se a distribuição do conteúdo dos artigos analisados segundo autores/ano de publicação, título, objetivo, periódico e região da pesquisa. $\mathrm{Na} 2^{\circ}$ etapa gerou-se um gráfico, caracterizando a categoria dos artigos e a porcentagem de cada categoria. Segue descrição no fluxograma abaixo (Figura1): 


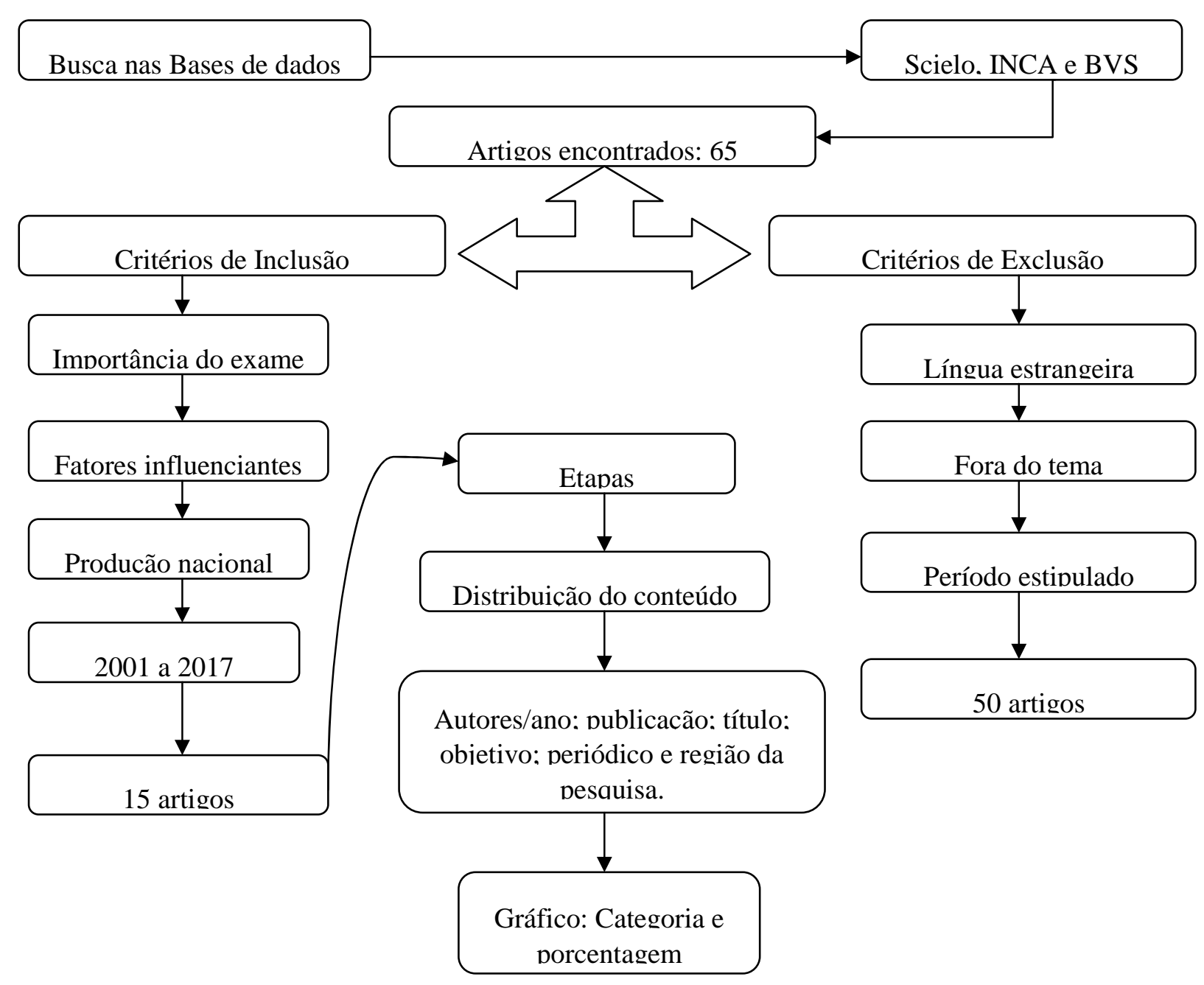

Figura 1- Fluxograma de demonstração da inclusão e exclusão dos artigos.

\section{RESULTADOS E DISCUSSÃO}

Conforme as diretrizes, é importante o profissional da atenção

primária

terceirizadas fazer as orientações frente as alterações citológicas Quadro 2.

Quadro 2 - Resumo de recomendações para conduta inicial frente aos resultados alterados de exames citopatológicos nas unidades de atenção básica

\begin{tabular}{|c|c|c|c|}
\hline \multirow{4}{*}{$\begin{array}{l}\text { Células } \\
\text { escamosas } \\
\text { atípicas de } \\
\text { significado } \\
\text { indeterminado } \\
\text { (ASCUS) }\end{array}$} & \multirow{3}{*}{$\begin{array}{l}\text { Possivelmente não } \\
\text { neoplásicas (ASC- } \\
\text { US) }\end{array}$} & $<25$ anos & Repetir em 3 anos \\
\hline & & $\begin{array}{l}\text { Entre } 25 \text { e } 29 \\
\text { anos }\end{array}$ & Repetir a citologia em 12 meses \\
\hline & & $\geq 30$ anos & Repetir a citologia em 6 meses \\
\hline & Não se podendo & & Encaminhar para colposcopia \\
\hline
\end{tabular}




\begin{tabular}{|c|c|c|c|}
\hline & $\begin{array}{l}\text { afastar lesão de alto } \\
\text { grau (ASC-H) }\end{array}$ & & \\
\hline $\begin{array}{l}\text { Células } \\
\text { glandulares } \\
\text { atípicas de } \\
\text { significado } \\
\text { indeterminado } \\
\text { (AGC) }\end{array}$ & $\begin{array}{l}\text { Possivelmente não } \\
\text { neoplásicas ou não } \\
\text { se podendo afastar } \\
\text { lesão de alto grau }\end{array}$ & & Encaminhar para colposcopia \\
\hline $\begin{array}{l}\text { Células atípicas } \\
\text { de origem } \\
\text { indefinida (AOI) }\end{array}$ & $\begin{array}{l}\text { Possivelmente não } \\
\text { neoplásicas ou não } \\
\text { se podendo afastar } \\
\text { lesão de alto grau }\end{array}$ & & Encaminhar para colposcopia \\
\hline \multirow{2}{*}{$\begin{array}{l}\text { Lesão de Baixo } \\
\text { Grau (LSIL) }\end{array}$} & & $<25$ anos & Repetir em 3 anos \\
\hline & & $\geq 25$ anos & Repetir a citologia em 6 meses \\
\hline $\begin{array}{l}\text { Lesão de Alto } \\
\text { Grau (HSIL) }\end{array}$ & & & Encaminhar para colposcopia \\
\hline $\begin{array}{l}\text { Lesão } \\
\text { intraepitelial de } \\
\text { alto grau não } \\
\text { podendo excluir } \\
\text { microinvasão }\end{array}$ & & & Encaminhar para colposcopia \\
\hline $\begin{array}{l}\text { Carcinoma } \\
\text { escamoso } \\
\text { invasor }\end{array}$ & & & Encaminhar para colposcopia \\
\hline $\begin{array}{l}\text { Adenocarcinoma } \\
\text { in situ (AIS) ou } \\
\text { invasor }\end{array}$ & & & Encaminhar para colposcopia \\
\hline
\end{tabular}

Fonte: BRASIL 2016

Dentro dos critérios de inclusão estabelecidos previamente, foram selecionados 10 artigos, dispostos no Quadro 3.

Quadro 3: Distribuição do conteúdo dos artigos analisados segundo autores/ano de publicação, título, objetivo, periódico e região da pesquisa.

\begin{tabular}{|c|c|c|c|c|c|}
\hline$N^{\mathbf{0}}$ & $\begin{array}{c}\text { Autores/Ano } \\
\text { de publicação }\end{array}$ & Título & Objetivo & Periódico & $\begin{array}{c}\text { Região } \\
\text { da } \\
\text { Pesquisa }\end{array}$ \\
\hline
\end{tabular}




\begin{tabular}{|c|c|c|c|c|c|}
\hline 1 & $\begin{array}{l}\text { KURMAN, R. } \\
\text { J; SOLOMON, } \\
\text { D. } 2001\end{array}$ & $\begin{array}{l}\mathrm{O} \text { sistema } \\
\text { Bethesda para o } \\
\text { relato de } \\
\text { diagnóstico } \\
\text { citológico } \\
\text { cervicovaginal. }\end{array}$ & $\begin{array}{lr}\text { Avaliar } & \text { e } \\
\text { atualizar } & \text { a } \\
\text { terminologia } & \text { do } \\
\text { Sistema Bethesda } \\
\text { de 1991 para } \\
\text { relatar } & \text { os } \\
\text { resultados } & \text { da } \\
\text { citologia cervical. }\end{array}$ & REVINTER & $\begin{array}{c}\text { Rio de } \\
\text { Janeiro - RJ }\end{array}$ \\
\hline 2 & $\begin{array}{c}\text { ROMAN, K. E. } \\
\text { M; PANIS, C. } \\
2010\end{array}$ & $\begin{array}{lr}\text { Identificação } & \text { dos } \\
\text { fatores de } & \text { risco } \\
\text { associados } & \text { ao } \\
\text { desenvolvimento } \\
\text { de câncer de } & \text { colo } \\
\text { uterino } & \text { em } \\
\text { mulheres. } & \end{array}$ & $\begin{array}{l}\text { Caracterizar uma } \\
\text { amostra de } \\
\text { mulheres em } \\
\text { relação à } \\
\text { probabilidade de } \\
\text { desenvolvimento } \\
\text { de câncer de colo } \\
\text { uterino através } \\
\text { dos fatores de } \\
\text { risco descritos } \\
\text { pela literatura. }\end{array}$ & Infarma & $\begin{array}{l}\text { Londrina - } \\
\text { PR }\end{array}$ \\
\hline 3 & $\begin{array}{l}\text { ZUQUE, F. R. } \\
\text { S, et al., } 2010\end{array}$ & $\begin{array}{l}\text { Citologia oncótica } \\
\text { durante a gestação: } \\
\text { desafio ou } \\
\text { realidade? }\end{array}$ & $\begin{array}{l}\text { Identificar as } \\
\text { dificuldades } \\
\text { vivenciadas pelos } \\
\text { enfermeiros para } \\
\text { realizar a coleta } \\
\text { do exame de } \\
\text { citologia oncótica } \\
\text { durante o período } \\
\text { gestacional. }\end{array}$ & Conexão & $\begin{array}{c}\text { Três } \\
\text { LagoasMS }\end{array}$ \\
\hline
\end{tabular}




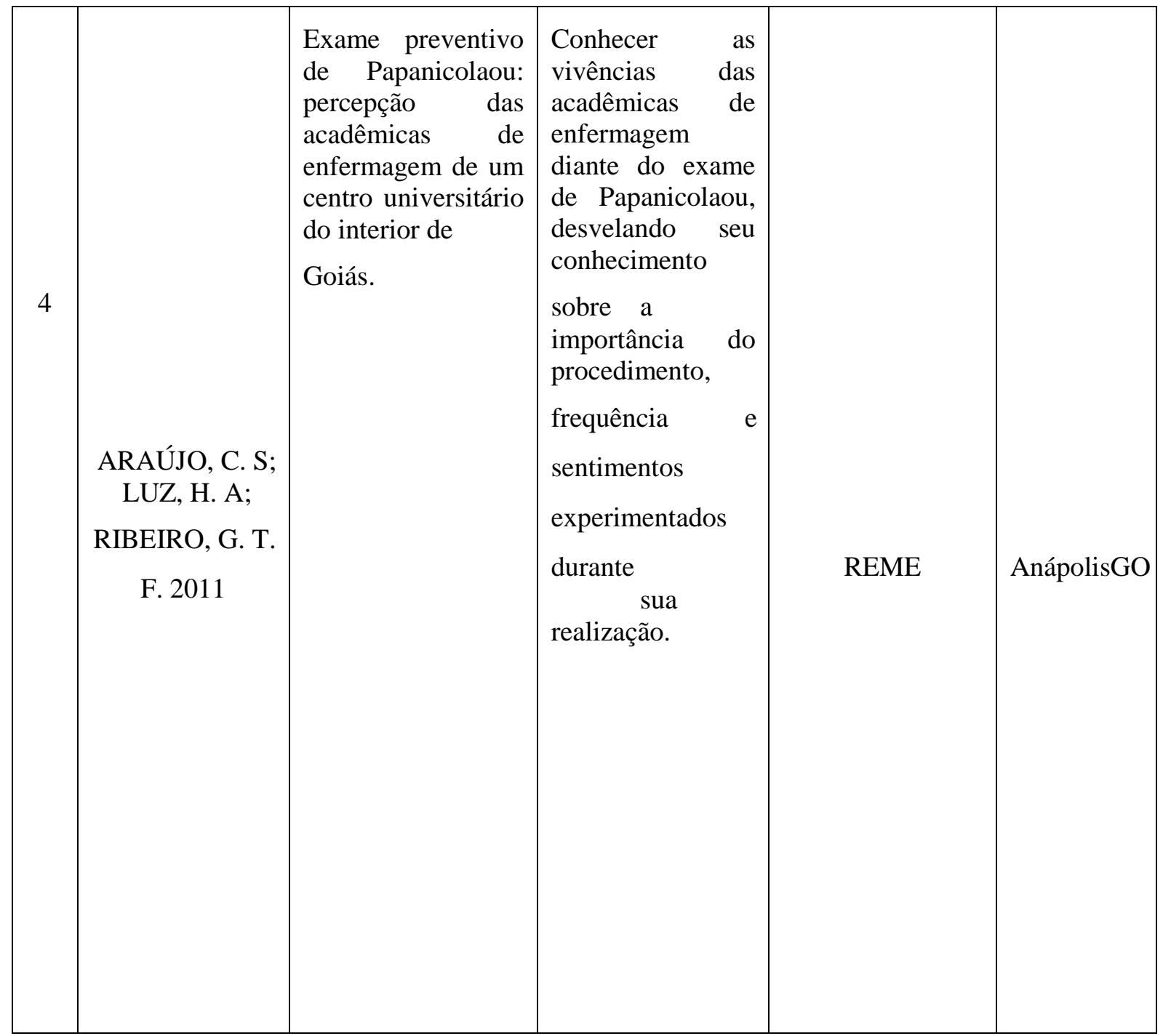

\begin{tabular}{|c|c|c|c|c|c|}
\hline 5 & $\begin{array}{l}\text { BATISTA, M. } \\
\text { L. S, et al., } 2012\end{array}$ & $\begin{array}{l}\text { Resultados } \\
\text { citopatológicos de } \\
\text { mulheres que } \\
\text { realizam exames do } \\
\text { colo do útero em } \\
\text { um laboratório } \\
\text { escola da } \\
\text { Universidade } \\
\text { Federal de Goiás, } \\
\text { Goiânia - GO: } \\
\text { estudo de } \\
\text { prevalência. }\end{array}$ & 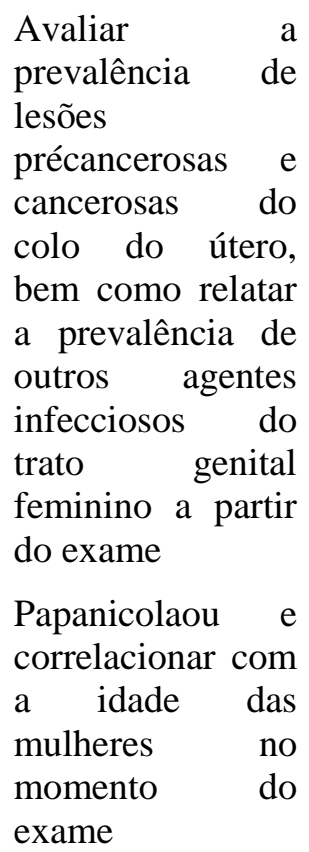 & $\begin{array}{c}\text { Revista do } \\
\text { Instituto de } \\
\text { Ciência da Saúde }\end{array}$ & $\begin{array}{c}\text { Goiânia - } \\
\text { GO }\end{array}$ \\
\hline
\end{tabular}




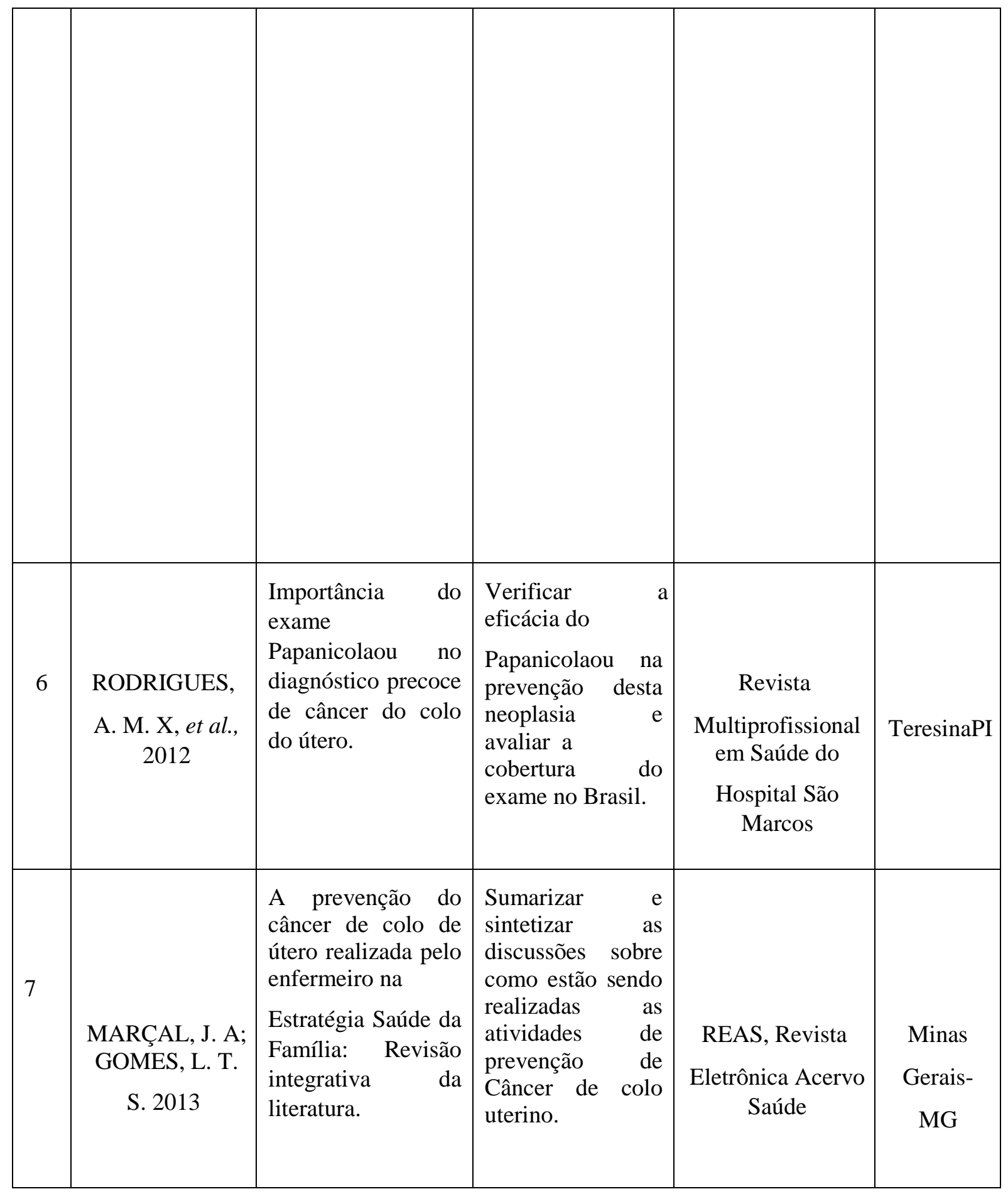




\begin{tabular}{|c|c|c|c|c|c|}
\hline 8 & $\begin{array}{c}\text { SANTANA, J. } \\
\text { E. O; SANTOS, } \\
\text { M; } \\
\text { MACHADO, I. } \\
\text { L. D. } 2013\end{array}$ & $\begin{array}{ll}\text { A importância } & \text { da } \\
\text { realização } & \text { do } \\
\text { Papanicolaou } & \text { em } \\
\text { gestantes: uma } & \\
\text { revisão de literatura }\end{array}$ & 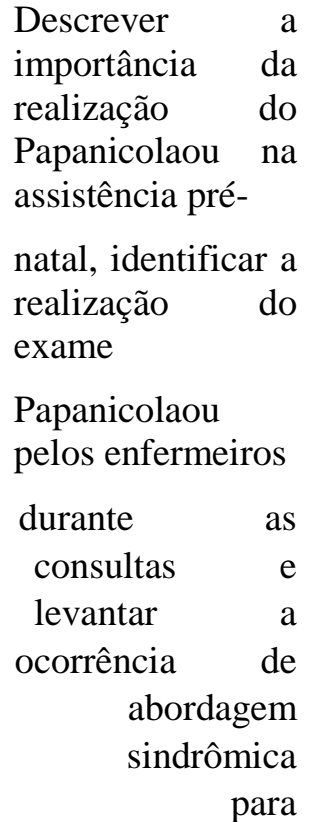 & $\begin{array}{c}\text { Revista de } \\
\text { Ciências } \\
\text { Biológicas e da } \\
\text { Saúde }\end{array}$ & AracajuSE \\
\hline
\end{tabular}

\begin{tabular}{|c|c|c|c|c|c|}
\hline & & & detecção de DST. & & \\
\hline 9 & $\begin{array}{l}\text { BARASUOL, } \\
\text { M. E. C; } \\
\text { SCHMIDT, D. } \\
\text { B. } 2014\end{array}$ & $\begin{array}{l}\text { Neoplasia do colo } \\
\text { do útero e seus } \\
\text { fatores de risco: } \\
\text { Revisão integrativa. }\end{array}$ & $\begin{array}{lr}\text { Avaliar } & \text { as } \\
\text { evidências } & \\
\text { disponíveis } & \text { na } \\
\text { literatura } & \text { a } \\
\text { respeito } & \text { dos } \\
\text { fatores de risco } \\
\text { para a neoplasia } \\
\text { de colo do útero. }\end{array}$ & $\begin{array}{l}\text { Revista Saúde e } \\
\text { Desenvolvimento }\end{array}$ & $\begin{array}{l}\text { Rio } \\
\text { Grande do } \\
\text { Sul-RS }\end{array}$ \\
\hline 10 & $\begin{array}{l}\text { NÓBREGA, A. } \\
\text { L, et al., } 2014\end{array}$ & $\begin{array}{l}\text { Importância da } \\
\text { assistência de } \\
\text { enfermagem para a } \\
\text { realização do } \\
\text { exame } \\
\text { citopatológico: um } \\
\text { olhar bibliográfico. }\end{array}$ & $\begin{array}{l}\text { Averiguar a } \\
\text { importância do } \\
\text { exame } \\
\text { citopatológico na } \\
\text { prevenção do } \\
\text { câncer de colo de } \\
\text { útero. }\end{array}$ & INTESA & PombalPB \\
\hline
\end{tabular}




\begin{tabular}{|c|c|c|c|c|c|}
\hline 11 & $\begin{array}{l}\text { SANTOS, M. } \\
\text { A, et al., } 2014\end{array}$ & $\begin{array}{l}\text { A importância da } \\
\text { prevenção do } \\
\text { câncer do colo } \\
\text { uterino: em pauta o } \\
\text { exame de } \\
\text { Papanicolaou. }\end{array}$ & $\begin{array}{l}\text { Identificar na } \\
\text { literatura } \\
\text { conhecimento das } \\
\text { mulheres sobre a } \\
\text { importância da } \\
\text { realização } \\
\text { exame de } \\
\text { Papanicolaou para } \\
\text { a prevenção do } \\
\text { câncer de colo } \\
\text { uterino. }\end{array}$ & $\begin{array}{c}\text { Recien, Revista } \\
\text { Científica de } \\
\text { Enfermagem }\end{array}$ & $\begin{array}{c}\text { São } \\
\text { Paulo-SP }\end{array}$ \\
\hline 12 & $\begin{array}{l}\text { CARVALHO, } \\
\text { R. S, et al., } 2017\end{array}$ & \begin{tabular}{lr}
\multicolumn{2}{l}{ Perfil preventivo do } \\
câncer de & colo \\
uterino & em \\
trabalhadoras & da \\
enfermagem. &
\end{tabular} & $\begin{array}{lr}\text { Descrever o perfil } \\
\text { preventivo do } \\
\text { câncer de } & \text { colo } \\
\text { uterino } & \text { em } \\
\text { trabalhadoras } & \text { da } \\
\text { Enfermagem. } & \end{array}$ & $\begin{array}{l}\text { REUOL, Revista } \\
\text { de Enfermagem }\end{array}$ & Recife-CE \\
\hline 13 & $\begin{array}{l}\text { PRATES, S. F. } \\
\text { L, et al., } 2017\end{array}$ & $\begin{array}{l}\text { Uma revisão de } \\
\text { literatura sobre o } \\
\text { perfil das } \\
\text { portadoras de } \\
\text { câncer de útero e } \\
\text { colo do útero. }\end{array}$ & $\begin{array}{l}\text { Conhecer o perfil } \\
\text { das portadoras de } \\
\text { câncer de útero e } \\
\text { colo de útero de } \\
\text { acordo com a } \\
\text { literatura. }\end{array}$ & $\begin{array}{c}\text { REAS, Revista } \\
\text { Eletrônica Acervo } \\
\text { Saúde }\end{array}$ & $\begin{array}{c}\text { Minas } \\
\text { Gerais- } \\
\text { MG }\end{array}$ \\
\hline
\end{tabular}




\begin{tabular}{|c|c|c|c|c|c|}
\hline 14 & $\begin{array}{l}\text { BELTRAMI, M. } \\
\text { A. L, et al., } \\
2017\end{array}$ & $\begin{array}{l}\text { A mulher frente à } \\
\text { importância de } \\
\text { realização do } \\
\text { exame preventivo } \\
\text { do câncer de colo } \\
\text { uterino. }\end{array}$ & $\begin{array}{l}\text { Mensurar o nível } \\
\text { de conhecimento } \\
\text { e percepção de } \\
\text { mulheres sobre a } \\
\text { importância da } \\
\text { realização do } \\
\text { exame preventivo } \\
\text { do câncer de colo } \\
\text { do útero. }\end{array}$ & $\begin{array}{c}\text { Revista Cientifica } \\
\text { do ITPAC }\end{array}$ & $\begin{array}{c}\text { Porto } \\
\text { Nacional - } \\
\text { TO }\end{array}$ \\
\hline 15 & $\begin{array}{l}\text { SOUSA, M. A. } \\
\text { A. } 2017\end{array}$ & $\begin{array}{l}\text { Importância do } \\
\text { teste Papanicolaou } \\
\text { na detecção } \\
\text { precoce do câncer } \\
\text { de colo do útero. }\end{array}$ & $\begin{array}{l}\text { Descrever a } \\
\text { importância do } \\
\text { teste } \\
\text { Papanicolaou no } \\
\text { diagnóstico } \\
\text { precoce do câncer } \\
\text { de colo uterino. }\end{array}$ & $\begin{array}{c}\text { Journal of } \\
\text { Medicine and } \\
\text { Health Promotion. }\end{array}$ & $\begin{array}{l}\text { Patos - } \\
\text { PB }\end{array}$ \\
\hline
\end{tabular}

Partindo da análise dos artigos selecionados, os estudos foram divididos em quatro categorias, conforme Gráfico 1: 


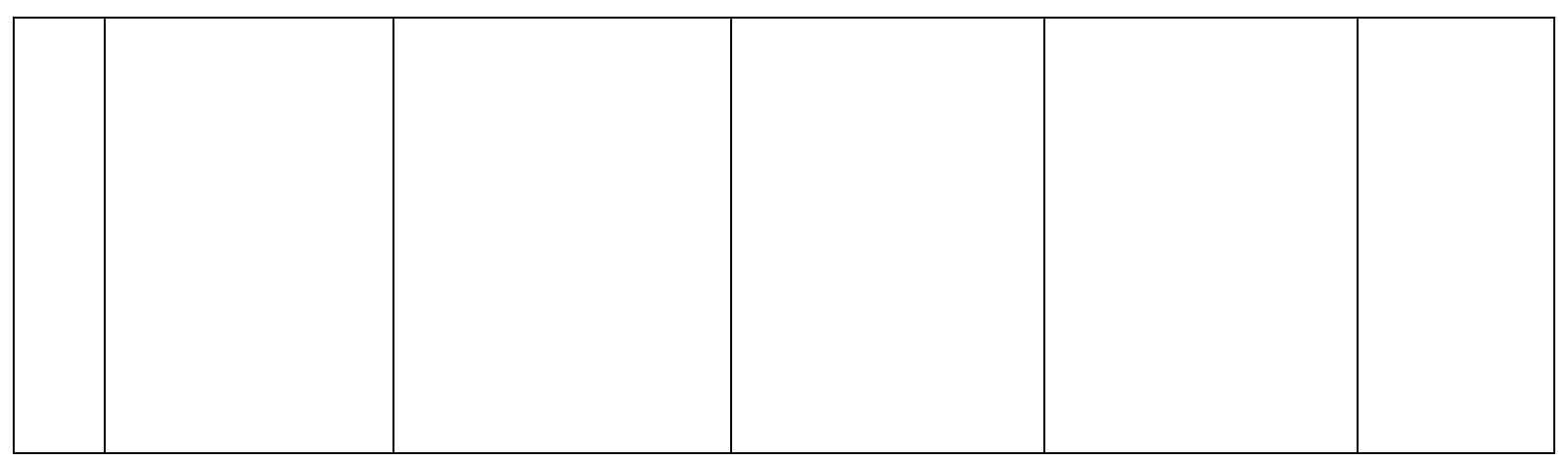

Gráfico 1: Distribuição dos Artigos em categorias e porcentagem

\section{ARTIGOS}

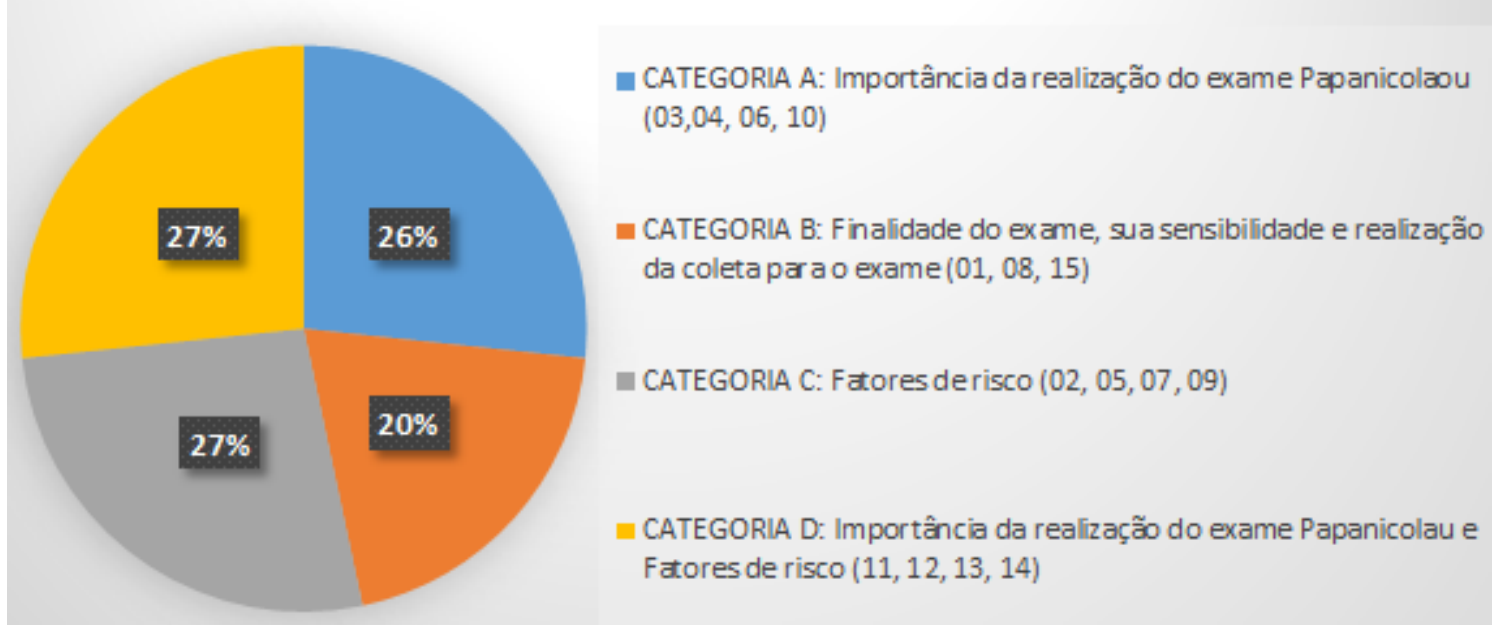

$\mathrm{Na}$ "Categoria A" os autores relatam sobre a importância da realização do exame Papanicolaou no diagnóstico precoce. Foram encontrados 4 artigos, sendo correspondentes a $1026 \%$ dos artigos selecionados. Seguem abaixo descrições:

Estudo realizado por Rodrigues et al. (2012) através de uma revisão de literatura sistemática, foi possível verificar que o exame preventivo Papanicolaou é de grande importância na detecção precoce das lesões que podem indicar uma suposta neoplasia uterina, pois diagnostica tanto alterações benignas como inflamações e processos de metaplasia escamosa, quanto malignas, como neoplasia intraepitelial cervical nos diversos graus. No estudo realizado por Nóbrega et al. (2014), através de uma revisão bibliográfica, ele destacou que o câncer de colo uterino é uma doença com alto índice de prevenção, tendo como método efetivo para o seu rastreamento o exame Papanicolaou. Contudo, a realização deste exame de prevenção permite reduzir em até $70 \%$ a mortalidade por câncer de colo de útero na 
população de risco, pois esta neoplasia tem um desenvolvimento lento, e as alterações celulares que podem desencadeá-la são facilmente descobertas no exame preventivo. Já no estudo realizado por Zuque et al. (2010), através de um levantamento bibliográfico, ele relatou sobre a importância da realização do exame Papanicolaou durante a gestação, pois o diagnóstico de câncer do colo uterino na gestação provoca um processo traumático para a gestante e seus familiares. Porém, com a realização da citologia durante a gestação, há possibilidade de detecção precoce do câncer e planejamento da conduta terapêutica adequada, o que proporcionará prognóstico favorável. Corroborando com os estudos acima, Araújo et al. (2011), na sua pesquisa descritiva, com abordagem qualitativa, confirma a importância do exame para a saúde da mulher, sendo possível detectar lesões pré-invasivas precocemente, e consequentemente, é instrumento essencial para a diminuição da mortalidade pelo câncer do colo uterino.

A "Categoria B" descreve a finalidade do exame, sua sensibilidade e a realização da coleta para o exame. Foram encontrados 3 artigos, correspondendo a $20 \%$ dos artigos selecionados. Os artigos relatam que:

Para realizar um diagnóstico precoce e eficaz deve-se realizar uma coleta correta e com qualidade, por isso Souza (2017), em seu artigo relata que o exame citológico Papanicolaou é um procedimento simples e barato. No qual é realizado um exame externo da vulva, em seguida, introduz o espéculo, também conhecido como bico de pato, pelo canal vaginal para permitir a visualização do colo do útero, onde é realizada a coleta e enviada para análise. $\mathrm{O}$ exame preventivo deve ser realizado por todas as mulheres sexualmente ativas ou não, pelo menos uma vez ao ano. No caso de pacientes grávidas, a coleta endocervical não é contraindicada, mas deve ser realizada de maneira cuidadosa e com uma correta explicação do procedimento e do pequeno sangramento que pode ocorrer após o procedimento. Como existe uma eversão fisiológica da junção escamo-colunar do colo do útero durante a gravidez, a realização exclusiva da coleta ectocervical na grande maioria destes casos fornece um esfregaço satisfatório para a análise laboratorial (SANTANA, SANTOS e MACHADO, 2013). De acordo com o relato do diagnóstico citológico - Bethesda (2001), quando negativo para lesão intraepitelial ou malignidade, apresentam: Alterações celulares reativas, podendo ser inflamação (incluindo reparo típico), atrofia com inflamação, radiação e outras. Também podem apresentar: Organismos como: Trichomonas vaginalis, fungos morfologicamente consistentes com Candida spp., desvio na flora sugestivo de vaginose bacteriana, bactérias morfologicamente consistentes com Actinomyces spp. e alterações celulares consistentes com 
vírus do herpes simples (KURMAN, SOLOMON, 2001). Com relação às células epiteliais anormais, estão inclusas as células escamosas e as glandulares. Nas células escamosas inclui: células escamosas atípicas de significado indeterminado (ASCUS), lesão intraepitelial escamosa de baixo grau (LSIL) que compreende: HPV, Displasia leve e NIC I e a lesão intraepitelial escamosa de alto grau (HSIL), compreende: Displasia moderada e acentuada, Carcinoma “in situ”, NIC II e III e Carcinoma de células escamosas. Já nas células glandulares está incluso: Células glandulares atípicas de significado indeterminado (AGUS), adenocarcinoma “in situ” e adenocarcinoma (KURMAN, SOLOMON, 2001). Quando o exame é realizado adequadamente, apresenta uma sensibilidade e especificidade de $77,8 \%$ e $100 \%$, demonstrando que o Papanicolaou é o método de triagem rentável para a detecção precoce de lesões cervicais, pré-malignas e malignas (SOUZA, 2017).

$\mathrm{Na}$ “Categoria C" são apontados os fatores de risco, sendo encontrados 4 artigos, o que corresponde a $27 \%$ dos artigos selecionados. Neles os autores relatam os principais fatores predisponentes para o desenvolvimento do câncer do colo uterino:

Os autores Roman e Panis (2010), realizaram um estudo tipo coorte-transversal, com componentes descritivos e comparativos, através de uma amostra aleatória da população de mulheres sexualmente ativas, das quais 55,4\% possuem segundo grau de escolaridade completo, estudo realizado na cidade de Londrina-PR. Nesse estudo observou-se que $60 \%$ do total das entrevistadas relataram algum fator de risco para o desenvolvimento de câncer uterino. Os principais fatores de risco relatados foram a não realização do exame preventivo de Papanicolaou anualmente (60\%); uso de contraceptivos hormonais (60\%); antecedentes de DSTs/problemas ginecológicos (52,5\%); antecedentes familiares de câncer uterino (7,5\%); tabagismo (17\%); sedentarismo (85\%), e maus hábitos alimentares (92,5\%). Através desse estudo observou que, embora o nível de escolaridade das entrevistadas seja elevado, existe a falta de conscientização e informação sobre os inúmeros fatores de risco e de que o câncer uterino pode ser prevenido através da realização anual do exame de Papanicolaou. As autoras Marçal e Gomes (2013), que realizaram uma revisão integrativa da literatura, observaram que os resultados dos artigos selecionados condizem com os dados encontrados na literatura em relação aos fatores de risco para o câncer de colo uterino. Elas relatam que além do HPV, existem outros fatores que contribuem para a etiologia deste tumor, como o tabagismo, baixa ingestão de vitaminas, multiplicidade de parceiros sexuais, iniciação sexual precoce e uso de contraceptivos orais. No estudo de Barasuol e Schmidt (2014), que trata de uma revisão integrativa da literatura, no qual analisaram os fatores de risco predisponentes para o 
desenvolvimento do câncer do colo uterino, eles relataram que é estimada a redução de cerca de $80 \%$ da mortalidade pelo câncer do colo do útero quando houver o rastreamento adequado das mulheres entre 25 a 64 anos de idade, bem como quando há tratamento precoce de lesões precursoras com alto potencial de malignidade ou carcinoma in situ. Relatam também que o HPV está presente na maioria dos cânceres do colo uterino, que uma estratégia importante no controle desse vírus é a vacina que protege contra os quatro principais tipos virais, sendo estes: 6, 11, 16 e 18. Corroborando com os estudos acima, Batista (2012), no seu estudo retrospectivo descritivo, relatou que a maior prevalência de lesões cancerosase pré-cancerosas encontram-se em mulheres jovens, com menos de 40 anos de idade. Devido à associação de fatores como o grande número de parceiros, início precoce da vida sexual ligado diretamente à falta do uso de preservativos e, consequentemente, infecção por doenças sexualmente transmissíveis como o HPV. O Papilomavírus humano (HPV) é considerado o agente etiológico central envolvido na gênese dos tumores cervicais, detectado em 99,7\% dos casos de câncer do colo do útero. O HPV também é encontrado em mais de $85 \%$ das neoplasias intraepiteliais cervicais de alto grau, consideradas precursoras do câncer do colo do útero. Com relação ao tabagismo, ao avaliarem o índice de dependência da nicotina em relação aos fatores de risco para o câncer do colo do útero, observaram que quanto maior o nível de dependência da nicotina, maior a suscetibilidade para o desenvolvimento de lesões cancerígenas (BARASUOL E SCHMIDT, 2014). Nesse mesmo estudo eles também relataram que a faixa etária mais acometida pela neoplasia do colo uterino é de 25 a 60 anos. As jovens vão se tornando população vulnerável justamente pelo início da vida sexual precoce, devido deixá-las mais perto de agravos relacionados à saúde reprodutiva e sexual (BARASUOL E SCHMIDT, 2014).

$\mathrm{Na}$ “Categoria D” foram correlacionados a importância da realização do exame papanicolaou e fatores de risco. Foram encontrados 4 artigos que relatam sobre as duas categorias, correspondendo a $27 \%$ dos artigos:

Estudos realizados por Santos et al. (2014), que relataram que o exame citopatológico é uma estratégia de rastreamento preconizado pelo Ministério da Saúde, devendo ser priorizado para mulheres entre 25 a 59 anos, estimam que uma redução de $80 \%$ da mortalidade por este tipo de câncer pode ser alcançada quando o rastreamento é realizado em mulheres na faixa etária de 25 a 65 anos, com o teste Papanicolaou. Esse exame de prevenção do câncer cérvico uterino, além de sua importância para a saúde da mulher, é um procedimento importante de detecção precoce de lesões pré-invasivas e, consequentemente, 
instrumento essencial para a diminuição da mortalidade por essa patologia. No estudo descritivo, exploratório, com abordagem quantitativa, desenvolvido no Hospital e Maternidade Sinhá Carneiro, no município de Santa Luzia/PB, com uma população de 34 profissionais de Enfermagem, percebeu-se que a maioria das profissionais da amostra se encontrava na faixa etária considerada de maior risco para o desenvolvimento do câncer do colo uterino. Sendo sua incidência aumentada nas mulheres com idades entre 30 e 39 anos. Antes dos 25 anos de idade prevalecem às infecções por HPV e lesões de baixo grau, que na maioria dos casos regride espontaneamente (CARVALHO et al., 2017). Prates et al. (2017), realizou uma revisão integrativa da literatura sobre a caracterização das portadoras de câncer de útero e colo do útero, evidenciando a partir desses artigos, no que se refere à idade, que o câncer do colo do útero se manifesta a partir dos 20 anos, sendo seu maior risco de 50 anos a 60 anos, porém as mulheres mais jovens, que iniciaram o tratamento mais precocemente obtiveram um resultado melhor, aumentando sua taxa de sobrevida. O diagnóstico prévio do câncer de colo uterino por meio do Papanicolaou junto ao tratamento da lesão local diminui cerca de $90 \%$ dos casos de câncer, porém essa queda de taxa de morbimortalidade depende do rastreamento realizado na população feminina de 25 a 64 anos. Corroborando com os estudos, Beltrami et al. (2017), desenvolveu uma pesquisa em uma Unidade Básica de Saúde em Porto Nacional - TO, composta por 30 mulheres, escolhidas de forma randomizada, no período de setembro e outubro de 2013. Nesse estudo ele destacou sobre a faixa etária e sobre o tabagismo. A faixa etária das entrevistadas estava no intervalo de 20 a 49 anos, período da vida das mulheres em que ocorre o aumento progressivo dos riscos para o desenvolvimento dessa neoplasia, passando a fazer parte da população alvo de prevenção do câncer uterino. Porém, diante da iniciação sexual precoce da população jovem, sugere-se atenção especial para jovens entre os 10 e 19 anos. Ainda sobre os fatores que predispõem ao câncer, a maioria das pacientes entre 30 e 39 anos relataram ser tabagistas.

\section{CONCLUSÕES}

O câncer do colo do útero se constitui num importante problema de saúde pública a nível mundial, com altos impactos nos países em desenvolvimento, já que $80 \%$ dos casos se concentram nestes países. Sendo assim, o Brasil está entre os países que compartilham de elevada incidência e mortalidade por esta neoplasia. Contudo, o câncer do colo do útero tem uma história natural bem conhecida, evolução lenta e fácil diagnóstico, o que permite a 
identificação das lesões precursoras da doença e chance de cura em quase $100 \%$ dos casos, se diagnosticado precocemente. O diagnóstico precoce é feito a partir da realização do exame preventivo, estratégia adotada oficialmente pelo Ministério da Saúde, que deve ser ofertado a todas as mulheres que já tenham iniciado a vida sexual.

O Papanicolaou é considerado a forma mais eficiente para se rastrear o câncer de colo uterino, mas apesar de possuir uma metodologia fácil, rápida e de baixo custo, os programas de rastreio e combate no Brasil não vêm atingindo as metas esperadas. Com base nos dados da revisão, percebeu-se uma estreita relação entre o comportamento sexual, contágio com agentes infecciosos e o desenvolvimento do câncer de colo uterino. O HPV é considerado como um fator de risco determinante para o desenvolvimento dessa neoplasia, porém, fatores como multiplicidade de parceiros, higiene íntima inadequada, tabagismo, alcoolismo e início precoce de atividade sexual vem se mostrando associados a um aumento no risco de se desenvolver o câncer de colo uterino.

Apesar de ter um alto potencial de prevenção e cura quando diagnosticado precocemente, ainda constitui importante desafio para a saúde pública no que se refere ao controle de sua elevada incidência e mortalidade no Brasil. A falta de conhecimento, a não realização do exame ou seguimento do tratamento, influenciam diretamente nos níveis de incidência do câncer do colo uterino.

Outro aspecto de suma importância é que o profissional da saúde tenha conhecimentos suficientes em relação à neoplasia do colo do útero, tanto em relação aos fatores de risco, importância do exame e qualidade do material coletado para realização do mesmo.

Observou-se um déficit nas publicações que relatam sobre a coleta de material para a realização do exame e sobre sua a sensibilidade, pois para garantir a qualidade dos exames coletados, é importante que os profissionais recebam educação continuada, acompanhando os estudos científicos e os novos achados em relação à citologia oncótica.

Contudo, presume-se uma redução de aproximadamente $80 \%$ na mortalidade por neoplasia do colo do útero apenas com o rastreamento de mulheres com idade entre 25 a 60 anos com o Papanicolaou e tratamento precoce de lesões precursoras ou carcinoma "in situ". Porém, é imprescindível que haja organização, integralidade e qualidade no rastreamento e acompanhamento dessas mulheres. Bem como a importância do profissional da saúde tenha conhecimentos suficientes em relação à neoplasia do colo do útero, tanto em relação aos 
fatores de risco, importância do exame e qualidade do material coletado para realização do mesmo, no diagnóstico precoce.

\section{REFERENNCIAS}

ARAÚJO, C. S; LUZ, H. A; RIBEIRO, G. T. F. Exame preventivo de Papanicolaou:

percepção das acadêmicas de enfermagem de um centro universitário do interior de Goiás.

REME-Revista Mineira de Enfermagem/vol. 15.3, 2316-9389, 2011. Disponível em: 〈http://www.reme.org.br/artigo/detalhes/48> Acesso: 03 Janeiro de 2019.

BARASUOL, M. E. C; SCHMIDT, D. B. Neoplasia do colo do útero e seus fatores de risco: Revisão Integrativa. Revista Saúde e desenvolvimento/vol. 6, n.3, Jul/Dez, 2014. Disponível em:

$<$ https://www.uninter.com/revistasaude/index.php/saudeDesenvolvimento/article/viewFile/31 2/228> Acesso em: 15 Abril de 2018.

BATISTA, M. L. S. et al. Resultados citopatológicos de mulheres que realizam exame do colo do útero em um laboratório escola da Universidade Federal de Goiás, Goiânia - GO: estudo de prevalência. Revista do Instituto de Ciência da Saúde/ vol. 30 (3): 201-5, 2012. Disponível em:

Lhttps://www.unip.br/presencial/comunicacao/punlicacoes/ics/edicoes/2012/03_julset/V30_n 3_2012_p201a205.pdf> Acesso em: 03 Janeiro de 2019.

BELTRAMI, M. A. L. et al. A mulher frente à importância de realização do exame preventivo do câncer de colo uterino. Revista Científica do ITAPAC, Araguaína, v.10, n. 1, pág. 63, pub. 7, Fevereiro, 2017. Disponível em:

<https://assets.itpac.br/arquivos/revista/2017-2/Artigo-7-ok.pdf> Acesso em: 12 Abril de 2018.

BRASIL, INSTITUTO NACIONAL DO CÂNCER - INCA. Estimativas 2018: incidência de câncer no Brasil. Coordenação de Prevenção e Vigilância. - INCA. Rio de Janeiro, p. 3839, 2017. Disponível em: 〈http://www.inca.gov.br/estimativa/2018/estimativa-2018.pdf > Acesso em: 05 Abril de 2018.

CARVALHO, R. S. et al. Perfil preventivo do câncer do colo uterino em trabalhadoras da enfermagem. Rev. Enferm. UFPE online., Recife, 11 (6): 2257-63, Jun., 2017. Disponível 
em: 〈https://periodicos.ufpe.br/revistas/revistaenfermagem/article/viewFile/23385/19034> Acesso em: 02 Abril de 2018.

Diretrizes brasileiras para o rastreamento do câncer do colo do útero / Instituto Nacional de Câncer José Alencar Gomes da Silva. Coordenação de Prevenção e Vigilância. Divisão de Detecção Precoce e Apoio à Organização de Rede. - 2. ed. rev. atual. - Rio de Janeiro: INCA, 2016: Disponível em < http://www.citologiaclinica.org.br/site/pdf/documentos/diretrizespara-o-rastreamento-do-cancer-do-colo-do-utero_2016.pdf> Acesso em: 26/08/2019.

KURMAN, R. J; SOLOMON, D. O sistema Bethesda para o relato de diagnóstico citológico cervicovaginal. REVINTER, $2^{\circ}$ edição, Rio de Janeiro, 2001. Disponível em: $\langle$ http://www.citologiaclinica.org.br/site/pdf/documentos/inca-sessao-casosginecologicos.pdf $>$ Acesso em: 16 Abril de 2018.

MARÇAL, J. A; GOMES, L. T. S. A prevenção do câncer de colo de útero realizada pelo enfermeiro na Estratégia Saúde da Família: Revisão integrativa da literatura. REAS, Revista Eletrônica Acervo Saúde, vol. 5 (2), 474-4789, 2013. Disponível em:

〈https://www.acervosaude.com.br/doc/artigo_035.pdf > Acesso em: 26 Junho de 2018.

NÓBREGA, A. L. et al. Importância da assistência de enfermagem para a realização do exame citopatológico: um olhar bibliográfico. INTESA (Pombal - PB - Brasil) v. 8, n. 2, p. 01-08, dez.; 2014. Disponível em:

<https://www.gvaa.com.br/revista/index.php/INTESA/article/view/3175/2716> Acesso em: 07 Março de 2018.

PINHEIRO, D. M; FERREIRA, D. L. A. Prevenção de câncer cervical a longo prazo em instituições de atenção ao idoso. Rev. Enferm. UFPI. v.2, n. 1, 2013. Disponível em: 〈http://www.ojs.ufpi.br/index.php/reufpi/article/view/850/pdf >Acesso em: 07 Março de 2018.

PRATES, S. F. L. et al. Uma revisão de literatura integrativa sobre o perfil das portadoras de câncer de útero e colo do útero. REAS, Revista Eletrônica Acervo Saúde, vol. Sup. 6, E1E7, 2017. Disponível em: 〈https://www.acervosaude.com.br/doc/S-15_2017.pdf> Acesso em: 24 Junho de 2018.

RODRIGUES, A. M. X. et al. Importância do exame Papanicolaou no diagnóstico precoce de câncer do colo do útero. Revista Multiprofissional em Saúde do Hospital São Marcos, v. I, n.01, 2012. Disponível em: 
<https://www.ojs.saomarcos.or.br/ojs/index.php/cientifica/article/downoald/8/4> Acesso em: 12 Abril de 2018.

ROMAN, K. E. M; PANIS, C. Identificação dos fatores de rico associados ao desenvolvimento de câncer de colo uterino em mulheres. Infarma, v. 22, nº 7/8, 2010.

Disponível em:

$\leq$ http://revistas.cff.org.br/?journal=infarma\&page=article\&op=view\&path\%5B $\% 5 \mathrm{D}=88 \& p a t$

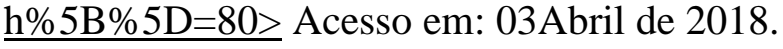

SANTANA, J. E. O; SANTOS, M; MACHADO, I. L. D. A importância da realização do papanicolaou em gestantes: uma revisão de literatura. Revista Ciências Biológicas e da Saúde, Aracajú - SE, v. 1, n.17, p. 39-48, out., 2013. Disponível em:

$\leq$ https://periodicos.set.edu.br/index.php/cadernobiologicas/article/view/581/534> Acesso em: 03 janeiro 2019.

SANTOS, M. A. et al. A importância da prevenção do câncer do colo uterino: em pauta o exame de Papanicolaou. Revista Recien, São Paulo. 4 (12): 15-20, 2014. Disponível em: 〈https://www.recien.com.br/index.php/Recien/article/view/78/144> Acesso em: 12 Abril de 2018.

SILVA, D. S. M. D. et al. Rastreamento do câncer do colo do útero no Estado do Maranhão, Brasil. Ciência \& Saúde Coletiva, v.19, n.4, 2014. Disponível em:

<http://www.cienciaesaudecoletiva.com.br/artigos/rastreamento-do-cancer-do-colo-douterono-estado-do-maranhao-brasil/12802?id=12802> Acesso em: 07 Agosto de 2018.

SOARES, M. B. O; SILVA, S. R. Intervenções que favorecem a adesão ao exame de colpocitologia oncótica. Revisão integrativa. Rev. Brasileira Enfermagem, v. 69, n. 2, p.404-404. 2016. Disponível em: 〈http://www.scielo.br/pdf/reben/v69n2/0034-7167-reben6902-0404.pdf $>$ Acesso em: 20 Agosto de 2018.

SOUSA, M. A. A. Importância do teste Papanicolaou na detecção precoce do câncer de colo do útero. Journal of Medicine and Health Promotion, 2 (3): 609-614, 2017. Disponível em: 〈https://www.ojs.saomarcos.or.br/ojs/index.php/cientifica/article/downoald/8/4>Acesso em: 03 Setembro de 2018.

SOUZA, M. T; SILVA, M. D; CARVALHO, R. Revisão integrativa: o que é e como fazer. 
Einstein, 8 (1 Pt 1): 102-6, 2010. Disponível em:

$\leq$ http://www.scielo.br/pdf/eins/v8n1/pt 1679-4508-eins-8-1-0102.pdf $>$ Acesso em: 01 Agosto de 2018.

ZUQUE, F. R. S. et al. Citologia oncótica durante a gestação: Desafio ou realidade? Revista Conexão, Três Lagoas, MS/vol.13, n.1 - 2010. Disponível

$\mathrm{em}: \leq \mathrm{http} / / /$ revistaconexão.aems.edu.br/wp_content/plugins/downloadattachments/includes/do wnload.php?id=1257> Acesso em: 03 janeiro 2019 